\title{
Case Report: A Polymicrobial Vision-Threatening Eye Infection Associated with Polysubstance Abuse
}

\author{
Venkata Satish Pendela, ${ }^{1 *}$ Pujitha Kudaravalli, ${ }^{2}$ Mamta Chhabria, ${ }^{1}$ and Emil Lesho ${ }^{1}$ \\ ${ }^{1}$ Rochester Regional Health, Rochester, New York; ${ }^{2}$ SUNY Upstate Medical University, Syracuse, New York
}

\begin{abstract}
We report a patient with risk factors for both microbial keratitis and endophthalmitis, which were initially challenging to distinguish. Cultures of corneal scrapings yielded several organisms, including an uncultivable Gramnegative rod, eventually identified as Kingella negevensis. Kingella negevensis is so named because most strains have been isolated in the Negev, a desert region of southern Israel. The epidemiology of $K$. negevensis remains incompletely understood. We found no other reports in the literature of this organism causing microbial keratitis.
\end{abstract}

\section{CASE REPORT}

A 38-year-old woman who uses intravenous drugs presented to the emergency department with right eye pain, redness, discharge, and blurry vision. She reports rubbing cocaine in her eyes when intoxicated. The patient had prior admissions for bacteremias. She had no known allergies, had not traveled outside the United States, and does not wear contact lenses. Physical examination revealed multiple needle marks along the veins in both upper and lower extremities; an $11 \times 11-m m$ corneal defect (Figure 1), with severe congestion of the conjunctiva; and a systolic ejection murmur in the aortic area. The patient was afebrile, and the total leukocyte count was $9,200 /$ LL. Blood cultures and a HIV assay were negative. The posterior chamber of the eye was not well visualized during slit-lamp examination because of the dense opacity of the corneal defect (Figure 1).

Before any anti-infectives were administered, two sets of stains and cultures were sent for routine bacterial, acid-fast, and fungal organisms. These were 1) corneal scrapings and 2) swabs of corneal surface and the eye discharge. Specimens were sent in aerobic and anaerobic nutrient transport media. Direct staining along with plating on sheep's blood, chocolate MacConkey, and Thayer-Martin agars was performed. The plates were aerobically and anaerobically incubated at $37^{\circ} \mathrm{C}$ in $5 \% \mathrm{CO}_{2}$. These grew Streptococcus parasanguinis, Propionibacterium acnes, and an unidentifiable Gram-negative rod (GNR) (Figures 2 and 3). She was treated topically with voriconazole, vancomycin, tobramycin, and prednisone eye drops, and systemically with ceftazidime and vancomycin. The need for intravitreal sampling and instillation of antibiotics was debated.

When initial cultures did not grow the GNR, repeated anaerobic and aerobic subculturing on various agars and broths including charcoal yeast extract, Columbia, and serum supplemented media was also performed.

Culturing on blood agar with vancomycin medium eventually grew Kingella spp. (catalase negative, oxidase positive, and acidification from glucose). Broad range bacterial qPCR and sequencing of a segment of 16S rRNA gene targeting groEL from this subculture matched to Kingella (genus level) and was most closely related to Kingella negevensis (species

\footnotetext{
*Address correspondence to Venkata Satish Pendela, Department of Internal Medicine, Rochester General Hospital, 1425 Portland Ave., Rochester, NY 14621. E-mails: pvsatish.135@gmail.com or satish. pendela@rochesterregional.org
}

level). The data were analyzed by a software called SmartGene (Lausanne, Switzerland). Systemic antibiotics were switched to linezolid and moxifloxacin. To help prevent corneal perforation, doxycycline and ascorbic acid were added. The patient was referred for a corneal transplant.

\section{DISCUSSION}

Although bacteremia, endocarditis, and hematogenous osteomyelitis are well-known complications of intravenous drug use, reports of ocular complications from applying the drugs topically to the surface of the eye are rare.

This case is notable for three reasons. First, the patient was at high risk for both types of endophthalmitis, endogenous (commonly associated with intravenous drug use) and exogenous or post-traumatic (from rubbing cocaine in his/her eyes). It was initially difficult to determine whether she had either or both types of endophthalmitis. Symptoms of endophthalmitis include pain and decreased visual acuity. Exudative lesions are found in the choroid and retina with vitreous haziness. Patients with exogenous endophthalmitis usually do not have fever or other signs of systemic infection when they have ocular symptoms. Blood cultures are usually negative, and the leukocyte count is normal or only minimally elevated. Absence of fever and negative blood cultures can be seen in endogenous cases as well. Therefore, diagnosis requires a high index of suspicion and, often times, vitreous sampling. ${ }^{1}$ Candida species are the most common pathogens followed by Aspergillus species.

Gram stains are positive in $40-50 \%$ of bacterial cases, and positive Gram stains are highly predictive of positive cultures. Negative stains do not have a good predictive value and do not rule out the diagnosis. If a Gram stain is performed on vitreous samples, care must be taken not to mistake what are known as pigment granules for Gram-positive cocci. Pigment granules are thought to be caused by the release of melanin from the iris or retinal pigmented endothelium. They appear sphenoidal or elliptical and can be distinguished because they appear to change color with changing the focus, whereas bacteria do not. ${ }^{1}$

The second notable teaching point is that although systemic antibiotics are used to treat underlying bacteremia or endocarditis, they alone are not sufficient for treating endophthalmitis. Direct injection of antibiotics into the vitreous is usually required. ${ }^{1}$ Because the antibiotics persist in the vitreous for only 1-2 days, repeat injections may be required, 


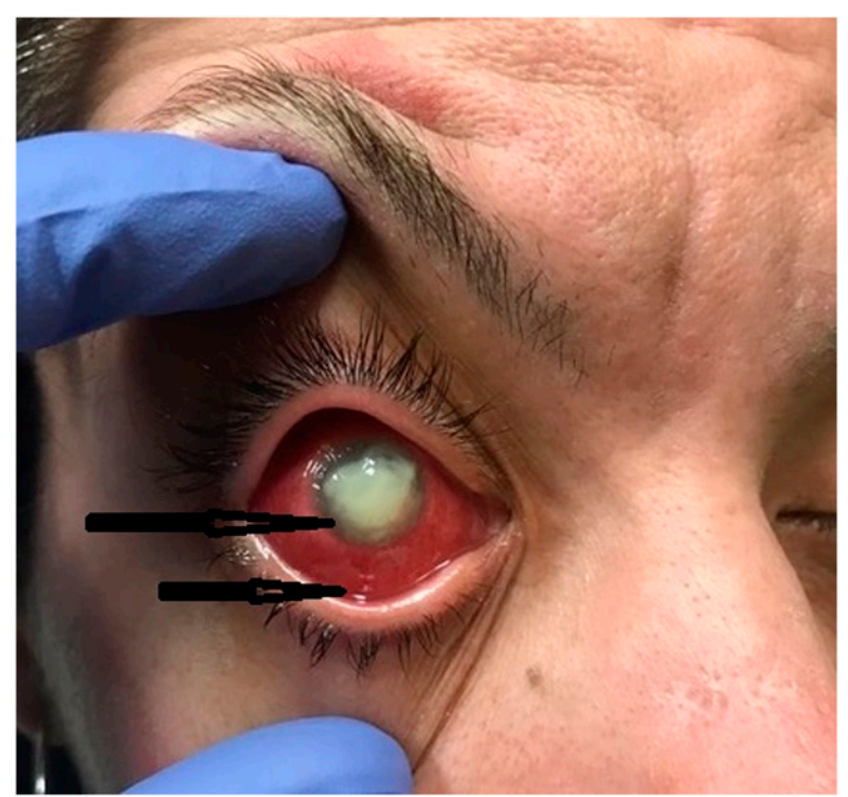

Figure 1. Corneal defect with severe eye congestion at presentation. Arrows pointing to an $11 \times 11-\mathrm{mm}$ corneal defect and surrounding conjunctivitis. This figure appears in color at www. ajtmh.org.

along with vitrectomy. ${ }^{1}$ As adjunctive therapy, oral antibiotics which are useful include linezolid and moxifloxacin because both produce therapeutic vitreous levels even in non-inflamed eyes. ${ }^{2}$ Oral doxycycline is used not to treat specific bacteria, but rather to prevent scarring and perforation. ${ }^{3}$ In this case,

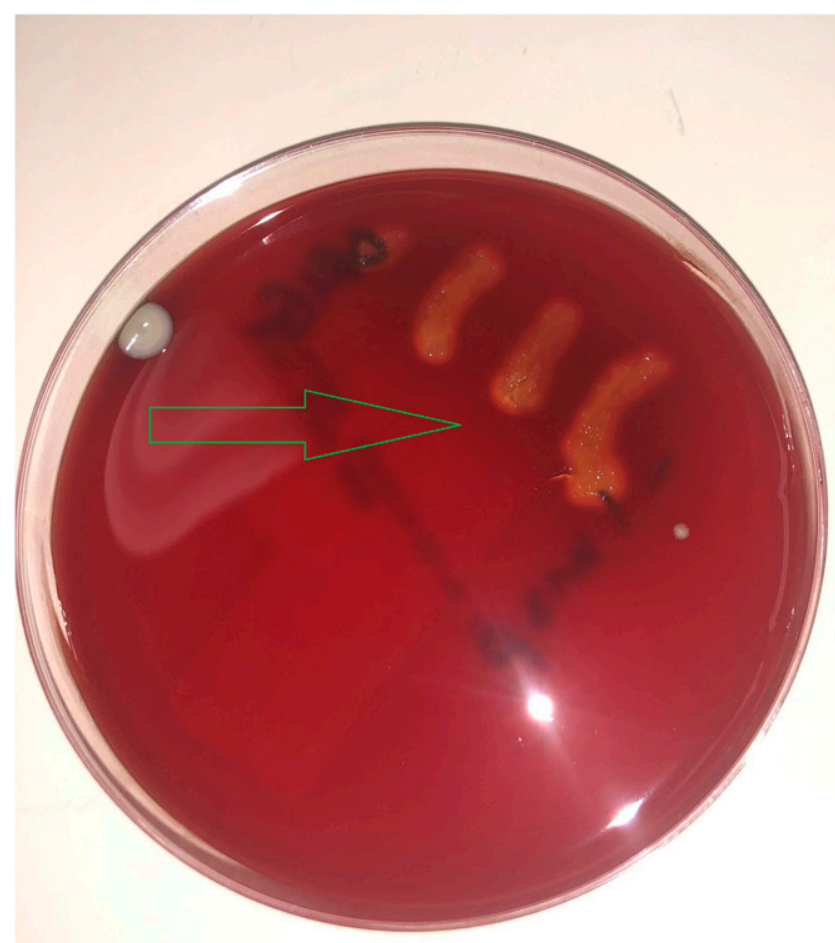

FIgURE 2. Microbial growth on a culture plate. Arrow pointing at polymicrobial growth. This figure appears in color at www.ajtmh.org.

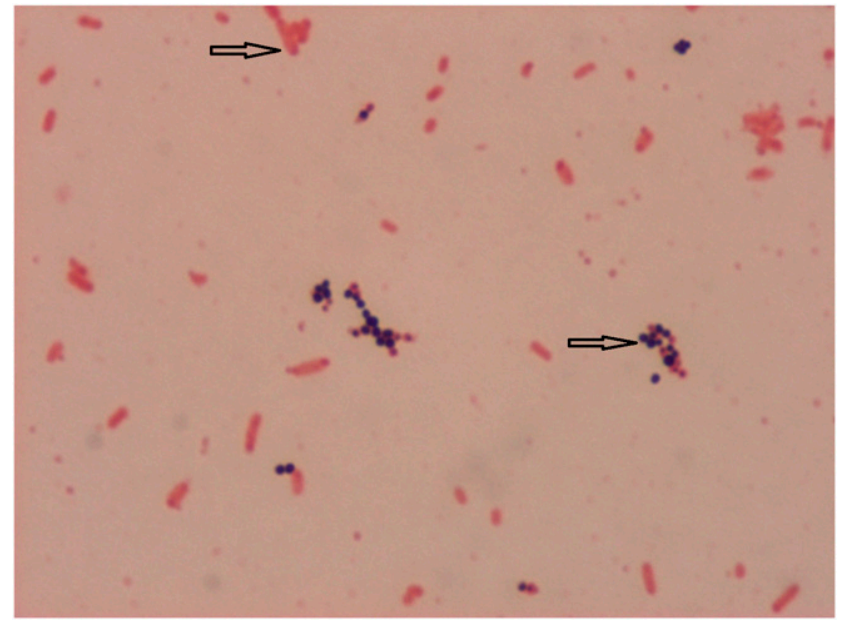

FIGURE 3. Gram stain of the corneal culture. Arrows pointing at Gram-positive cocci and Gram-negative rods. This figure appears in color at www.ajtmh.org.

there was a debate about treating with intravitreal antibiotics and/or vitrectomy, as post-traumatic endophthalmitis could not be initially ruled out. Subsequently, by day 4, the posterior chamber was visible and endophthalmitis was ruled out. Therefore, we continued with systemic antibiotics, followed by long-term doxycycline, while the patient awaited a corneal transplant.

Even though this patient ultimately did not have endophthalmitis, microbial keratitis is also a vision-threatening condition. Causes of microbial keratitis include Gram-negative organisms such as Pseudomonas aeruginosa; Gram-positive organisms such as Staphylococcal and Streptococcal species; viruses such as herpes simplex, varicella, and adenovirus; and other organisms such as Acanthamoeba. Symptoms are similar to those of endophthalmitis and include severe pain, decreased vision, and photophobia. Microbial keratitis is usually treated with fortified topical antibiotics-usually fortified aminoglycosides and/or cefazolin. Of note, topical antibiotics in the solution form penetrate ocular tissues better than ointment forms, even though patients might prefer ointments for ease of delivery and comfort.

The third notable aspect of this case is that initially the identity and susceptibility of the Gram-negative organism isolated from the corneal culture could not be defined, even with repeated subculturing on specialized nutrient media. The ongoing requirement for topical antibiotics unavoidably hampers subsequent ability to isolate and identify organisms from corneal samples. Identification ultimately required $16 \mathrm{~S}$ rRNA sequencing, which disclosed $K$. negevensis, a novel species cultivated from the oropharynx of children. ${ }^{4}$ Kingella negevensis is so named because most strains have been isolated in the Negev, a desert region of southern Israel. ${ }^{4,5}$ The epidemiology of $K$. negevensis remains largely unknown. ${ }^{5}$ Kingella negevensis has been previously shown to exist in human organs under nonpathogenic conditions. ${ }^{6}$ Unfortunately, we did not culture the contralateral eye, as it exhibited no signs of infection. Kingella kingae is the commoner species which has clinical implications in the pediatric population, including bacteremia, endocarditis, and joint and bone infections. ${ }^{7,8}$ Extensive review of the literature showed 
only a few case reports of $K$. kingae eye infection. ${ }^{9}$ We found no reports of $K$. negevensis causing clinically significant infections.

Received March 18, 2020. Accepted for publication April 14, 2020.

Published online May 18, 2020.

Authors' addresses: Venkata Satish Pendela and Mamta Chhabria, Department of Internal Medicine, Rochester General Hospital, Rochester, NY, E-mails: pvsatish.135@gmail.com and mamta.chhabria@ rochesterregional.org. Pujitha Kudaravalli, Department of Internal Medicine, SUNY Upstate Medical University, Syracuse, NY, E-mail: pkudaravalli17@gmail.com. Emil Lesho, Department of Infectious Diseases, Rochester General Hospital, Rochester, NY, E-mail: emil.lesho@ rochesterregional.org.

\section{REFERENCES}

1. Mandell G, Dolin R, Bennett J, 2009. Mandell, Douglas, and Bennett's Principles and Practice of Infectious Diseases, 7th edition. Cambridge, UK: Elsevier.

2. Fiscella RG, Lai WW, Buerk B, Khan M, Rodvold KA, Pulido JS, Labib S, Shapiro MJ, Blair NP, 2004. Aqueous and vitreous penetration of linezolid (Zyvox) after oral administration. Ophthalmology 111: 1191-1195.

3. Smith VA, Cook SD, 2004. Doxycycline-a role in ocular surface repair. Br J Ophthalmol 88: 619-625.

4. El Houmami $\mathrm{N}$ et al., 2017. Isolation and characterization of Kingella negevensis sp. nov., a novel Kingella species detected in a healthy paediatric population. Int J Syst Evol Microbiol 67: 2370-2376.

5. Yagupsky P, El Houmami N, Fournier PE, 2018. Respiratory carriage of the novel Kingella negevensis species by young children. New Microbes New Infect 26: 59-62.

6. Opota O, Laurent S, Pillonel T, Léger M, Trachsel S, Prod'hom G, Jaton K, Greub G, 2017. Genomics of the new species Kingella negevensis: diagnostic issues and identification of a locus encoding a RTX toxin. Microbes Infect 19: 546-552.

7. Yagupsky $\mathrm{P}, 2004$. Kingella kingae: from medical rarity to an emerging paediatric pathogen. Lancet Infect Dis 4: 358-367.

8. El Houmami N et al., 2017. Molecular tests that target the RTX locus do not distinguish between Kingella kingae and the recently described Kingella negevensis species. J Clin Microbiol 55: 3113-3122.

9. Muñoz-Egea MC, García-Pedrazuela M, González-Pallarés I, Martínez-Pérez M, Fernández-Roblas R, Esteban J, 2013. Kingella kingae keratitis. J Clin Microbiol 51: 1627-1628. 